\title{
Study of Design Challenges and Potential Applications of Wireless Sensor Network
}

\author{
Samarth Saxena \\ Department of Electronics and \\ Communication Engineering \\ BBDITM, Lucknow
}

\author{
Nandani Raj \\ Department of Electronics and \\ Communication Engineering \\ BBDITM, Lucknow
}

\author{
Kalyan Krishna Awasthi \\ Research Scholar, Department of \\ Electronics and Communication \\ Engineering \\ BBD University, Lucknow
}

\begin{abstract}
Wireless Sensor Network has revolutionized the entire sensing technology worldwide. It has found immense application after the advancement of wireless network system. WSNs are composed several sensor nodes through appropriate routing protocols. This research work is focused on the design overview of WSN along with it multiple potential applications in various fields. This paper also covers the routing protocols of WSNs and the challenges faced by it. This paper includes the several sphere of this powerful technology in a significant way.
\end{abstract}

\section{Keywords}

Wireless Sensor Network

\section{INTRODUCTION}

Advancement in the field of communication has led to the development of wireless sensor network comprising of several sensor nodes. This network is infrastructure-less that is installed in a bulk (Ad-hoc manner) [1] for monitoring the system.

The integral part of this network is the power unit or the sensor nodes, which is basically a battery or a set of batteries, these nodes are used with onboard processor for managing \& monitoring the physical environment conditions of the concerned area. The nodes along with the processors are further connected to the base station which is center for the processing to take place, Base station is further connected to the internet for sharing data to the user concerned.

Hence, WSNs are connected with sensing, processing, analysis, storing and mining of data

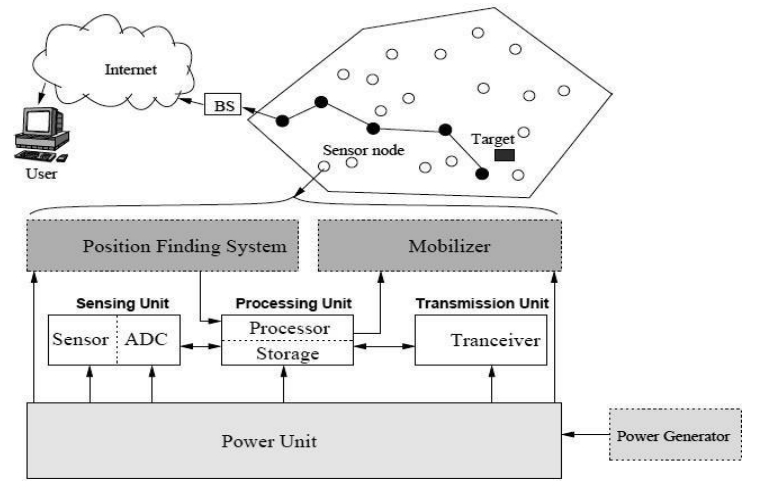

Fig.1. Architecture of Sensor Nodes (source [2])

Sensing unit senses the physical quantity which further transformed into digital through ADC. Thereafter processor carry out computations and transceiver transmit and receives data through other nodes connected to it. Power unit is the most significant part of any sensor once exhausted can't be replaced by other application dependent units like Mobilizer, Power Generation \& Location Finding System.

\section{WSN's Architecture}

The wireless sensor network commonly follows the OSI architecture model (i.e., open system interconnection). It is a reference model for various applications communicating over a single network.[3]

\subsection{Types Of Architecture}

There are two types of architecture in WSN, they are -:

1. Layered Network Architecture

2. Clustered Network Architecture

These Architecture find wide application in different places like hospitals, building as well as in different applications such as disaster management \& security management.

\subsection{Layered Network Architecture}

The WSN's has five layered architectures with three cross layers. These networks mostly require five layersnamely application, transport, network, datalink and physical layer. The three cross layers are power management, mobility management stacked together forming sink, making the network efficient and competent.[4]

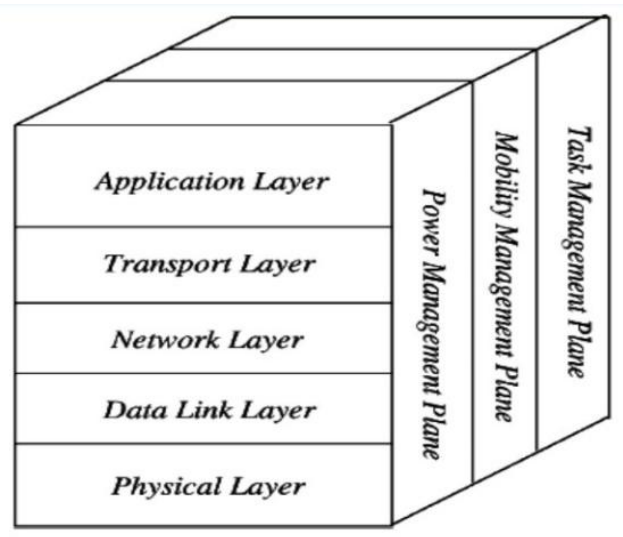

Fig.2. Protocol Stack used by sink (source [5])

These sensor networks architecture has several sensor nodes with its base station. The Architecture consisting of five layers and three cross layers forms a protocol stack. The application layer is liable for traffic management and offers software for numerous applications that convert the data in clear form to 
find positive information. The cross layer are concerned with network control and sensory function, overall improving its efficiency. The layered network system uses hundreds of similar nodes, these networks nodes are arranged into concentric layer.

\subsection{Clustered Network Architecture}

This architecture has group of sensor nodes called clusters that works according to "LEACH Protocol" [6,7]. This protocol stands for "Low Energy Adaptive clustering hierarchy". It uses the data fusion concept so that the network energy is efficient. In every cluster, each node could interact from the head of the cluster to get the required data. This approach selects nodes based on their residual energy content as the energy of all the constituent nodes are different. High energy nodes are used for processing and transmitting whereas low energy nodes are used for sensing purpose. [8,9]

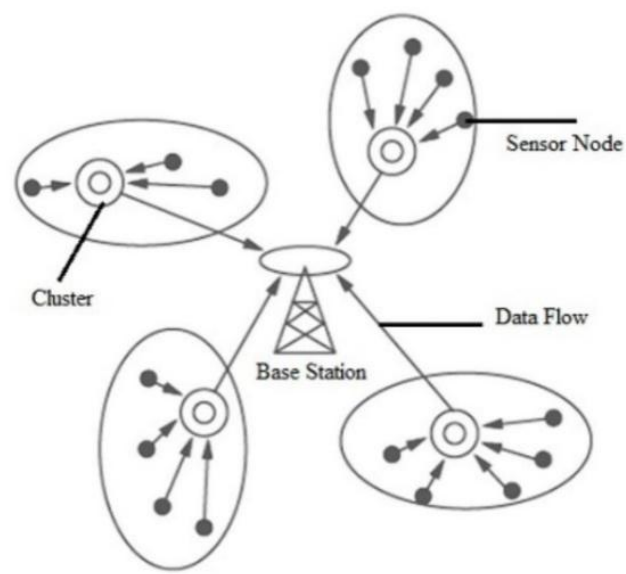

Fig.3. Architecture of Clustered Based Network [8]

\section{Design Challenges or Routing}

\section{Problems}

Design and Routing problems [10] depends on following Factors, and the Factors are: -

- $\quad$ Fault tolerance: -The failure of sensor nodes due to any cause should not affect the overall working of the network.

- Scalability: -No. of sensor nodes in a region may vary to extreme depending on application. The new scheme should able to cope with it.

- Production Cost: -Cost of each sensor node must be low as it would affect the cost of entire network.

- Hardware Constraints: -Design of the hardware must be energy efficient.

- Sensor Network Topology: -Several malfunctional nodes makes the network topology maintenance a coping with frequent challenging task.

For realization of such powerful network system various protocols and algorithm of networking techniques comes into force one of them is routing protocol

\section{ROUTING PROTOCOLS}

These are set of rules that defines how the manner in which several nodes will communicate among themselves \& how the data will be disseminated through the network. $[10,11]$

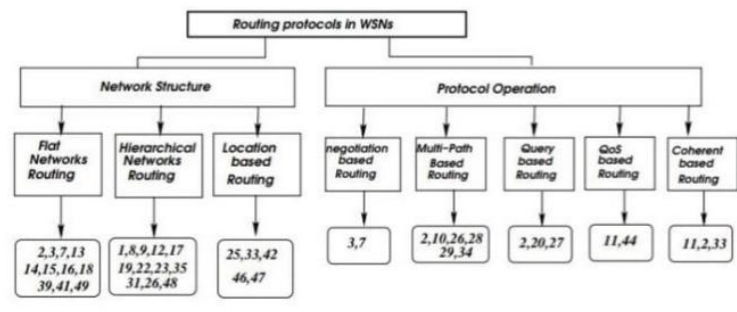

Fig.4 Classification of Routing Protocol [12]

\section{POTENTIAL SENSOR NETWORK APPLICATIONS}

There are wide variety of sensor that comes under the wireless sensor network such as seismic, acoustic, visual \& many more. These sensors are capable for monitoring various ambient conditions like,

- Temperature-: sensor node temperature are greatly influenced by the external and internal temperature, thereby worsening the lifetime of the network.[13]

- Pressure-: Several pressure sensors throughout the whole network are integrated to collect data. The real time pressure monitoring is designed based on WSNs technology.[14]

- Noise level -: Various real-life applications like, environmental noise measurement and acoustic event are monitored through novel system that utilizes the WSNs to sense.

- Mechanical stress level -: These sensors are based on resistive force whose electrical resistance is inversely related to the stress applied (elongation or pressure). They are sensitive to mechanical properties.

- Speed,Direction or Size of an object-: These types of sensors find wide applications to measure speed of engine,camshaft and wheels speed in auto mobile sector such as -: wind turbine, actuators etc. $[15,16]$

Micro-sensing and wireless connection together form a powerful connection for detection purposes.Hence, they find huge application in the area like military, environment, health

and other commercial fields. Its application could be categorized into the following,

- Area monitoring such as to detect enemy intrusion or oil pipelines.

- Health care monitoring includes continuous diagnosis of overall person's health.

- Habitat monitoring for keeping a check on various existing species around the globe like zebra in Kenya. 
- Threat detection, where wide area tracking system is used for detecting grounded nuclear bombs.

- Environmental / earth sensing for monitoring air pollutants, water temperature, forest fire detection etc.

WSNs have emerged as potentially promising alternatives in numerous fields, due to its characteristic features such as: -

- $\quad$ Real time monitoring

- Relatively low cost

- Unmanned operation

- Durability

- Self-organizing capabilities, etc.

\section{CONCLUSION}

WSN have been profoundly used in every aspect of human existence. The sensing technology has overcome various existing constraints and still a lot more to be overcome by further advancement. This paper has presented all the important aspects of WSNs, aiming to highlight its different attributes. It has large scope dealing different aspects of human existence. Many of research are currently going on developing better technologies needed for optimizing the system. Although WSN is promising technique, still numerous challenges are needed to be looked upon.

\section{REFERENCES}

[1] M.ChithikRaja, N.Mohamed Yasin,P. Ponnurajan“A Review on Ad-hoc Network Security"International Journal of Computer Applications Vol.1. No.6,2011

[2] K.K Awasthi, A.K Singh, Dr Shailendra Tahilyani "Study of Clustered Based Routing Protocol in WSN"International Journal of Computer Applications (0975 - 8887) Volume *No.*, 163(1):39-42, April 2017

[3] R.K Singh, Shweta Singh "Architecture of Wireless Network" International Journal of Computer Applications, Vol.9. No.2 march 2012.

[4] J. Agre, L. Clare, An integrated architecture for cooperative sensing networks, IEEE Computer Magazine (May 2000) 106-108.

[5] I.F. Akyildiz, W. Su*, Y. Sankar Subramaniam, E. Cayirci "Wireless sensor networks: a survey" Broadband and Wireless Networking Laboratory, School of Electrical and Computer Engineering, Georgia Institute of Technology, Atlanta, GA 30332, USA Received 12 December 2001; accepted 20 December 2001

[6] Rajesh Patel, Sunil Pariyani, Vijay Ukani," Energy and Throughput Analysis of Hierarchical Routing Protocol (LEACH) for Wireless Sensor Networks", International
Journal of Computer Applications Volume 20- No. 4 (April 2011).

[7] M. Bani Yassein, A. AL-zou'bi, Y. Khamayseh, W. Mardini, "Improvement on LEACH Protocol of Wireless Sensor Networks", International Journal of Digital Content Technology and its Applications Volume 3- No. 2 (June 2009).

[8] Amrinder Kaur, Sunil Saini," Simulation of Low Energy Adaptive Clustering Hierarchy Protocol for Wireless Sensor Network," International Journal of Advanced Research in Computer Science and Software Engineering, Volume 3, Issue7, July 2013.

[9] A.A. Abbasi, et al. A survey on clustering algorithms for wireless sensor networks ComputCommun, 30 (1415) (2007), pp. 2826-2841

[10] ParulKansal, Deepali KAnsal, Arun Balodi, "Compression of Various Routing Protocol in Wireless Sensor Networks", International Journal of Computer Applications Volume 5-No. 11(August 2010).

[11] Rajesh Patel, Sunil Pariyani, Vijay Ukani," Energy and throughput Analysis of Hierarchical Routing Protocol for Wireless Sensor Networks", International Journal of Computer Applications Volume 20- No. 4 (April 2011).

[12] Yuh Ren Tsai, "Coverage Preserving Routing Protocols for Randomly Distributed Wireless Sensor Networks", IEEE Transactions on Wireless Communications, Volume 6- No. 4 (April 2007).

[13] Boano, C.A., Tsiftes, N., Voigt, T., Brown, J. and Roedig, U., 2009. The impact of temperature on outdoor industrial sensor net applications. IEEE Transactions on Industrial Informatics, 6(3), pp.451-459

[14] Ahmad Khasawneh1*, Muhammad Shafie Bin Abd Latiff1, Hassan Chizari1,MoeenUddin Tariq1 and Abdullah Bamatrafl "PRESSURE BASED ROUTING PROTOCOL FOR UNDERWATER WIRELESS SENSOR NETWORKS: A SURVEY" KSII TRANSACTIONS ON INTERNET AND INFORMATION SYSTEMS VOL. 9, NO. 2, Feb. 2015.

[15] Miguel Torres-Ruiz1,Miltiadis D Lytras2,3 and Hassan Mathkour4" Innovative services and applications of wireless sensor networks: Research challenges and opportunities" International Journal of Distributed Sensor Networks 2018, Vol. 14(5).

[16] Mainwaring A, Culler D, Polastre J, et al. Wireless sensor networks for habitat monitoring. In: Proceedings of the 1st ACM international workshop on wireless sensor networks and applications, Atlanta, GA, 28 September 2002, pp.88-97. New York: ACM. 